\title{
The right ventricle in pulmonary arterial hypertension
}

\author{
Robert Naeije ${ }^{1}$ and Alessandra Manes ${ }^{2}$
}

Affiliations: 'Dept of Cardiology, Erasme University Hospital, Brussels, Belgium. ${ }^{2}$ Dept of Experimental, Diagnostic and Specialty Medicine (DIMES), Bologna University Hospital, Bologna, Italy.

Correspondence: Robert Naeije, Dept of Physiology, Faculty of Medicine, Free University of Brussels, Erasme Campus CP 640, 808 Route de Lennik, B-1070 Brussels, Belgium. E-mail: rnaeijedulb.ac.be

ABSTRACT Pulmonary arterial hypertension $(\mathrm{PAH})$ is a right heart failure syndrome. In early-stage PAH, the right ventricle tends to remain adapted to afterload with increased contractility and little or no increase in right heart chamber dimensions. However, less than optimal right ventricular (RV)-arterial coupling may already cause a decreased aerobic exercise capacity by limiting maximum cardiac output. In more advanced stages, RV systolic function cannot remain matched to afterload and dilatation of the right heart chamber progressively develops. In addition, diastolic dysfunction occurs due to myocardial fibrosis and sarcomeric stiffening. All these changes lead to limitation of RV flow output, increased right-sided filling pressures and under-filling of the left ventricle, with eventual decrease in systemic blood pressure and altered systolic ventricular interaction. These pathophysiological changes account for exertional dyspnoea and systemic venous congestion typical of $\mathrm{PAH}$. Complete evaluation of RV failure requires echocardiographic or magnetic resonance imaging, and right heart catheterisation measurements. Treatment of RV failure in PAH relies on: decreasing afterload with drugs targeting pulmonary circulation; fluid management to optimise ventricular diastolic interactions; and inotropic interventions to reverse cardiogenic shock. To date, there has been no report of the efficacy of drug treatments that specifically target the right ventricle.

0 @ERSpublications

The compensatory adaptation of right ventricular structure and function is linked to symptoms and prognosis in PAH http://ow.ly/D5W9R

\section{Introduction}

Case 1 was a 10-year-old boy was referred to a pulmonary hypertension centre after several episodes of exercise-induced syncope. A diagnosis of idiopathic pulmonary arterial hypertension (IPAH) was made following a step-by-step approach following current guidelines [1]. Right heart catheterisation showed a mean pulmonary artery pressure (mPAP) of $54 \mathrm{mmHg}$, and normal pulmonary capillary wedge pressure and cardiac output. There was no reversibility of increased mPAP and pulmonary vascular resistance (PVR) with inhaled nitric oxide. He was treated with an endothelin receptor antagonist (ERA) and acenocoumarol. Syncope did not recur. 8 years later, at the age of 18 years, he was in World Health Organization (WHO) functional class I and walked $580 \mathrm{~m}$ in $6 \mathrm{~min}$. A cardiopulmonary exercise test (CPET) showed a peak oxygen uptake $\left(V^{\prime} \mathrm{O}_{2}\right.$ peak $)$ of $39.9 \mathrm{~mL} \cdot \mathrm{kg}^{-1} \cdot \mathrm{min}^{-1}(86 \%$ predicted) and a ventilatory equivalent for minute ventilation $\left(V^{\prime} \mathrm{E}\right) /$ carbon dioxide production $\left(V^{\prime} \mathrm{CO}_{2}\right)$ of 27 . Right heart catheterisation showed a persistently high mPAP of $42 \mathrm{mmHg}$ with normal cardiac output and normal pulmonary capillary wedge pressure. Doppler echocardiography showed a maximum velocity of tricuspid regurgitation (TRV) of $4 \mathrm{~m} \cdot \mathrm{s}^{-1}$, allowing for the estimation of $\mathrm{mPAP}$ at $46 \mathrm{mmHg}$, normal right ventricular (RV) dimensions, a

Received: Aug 292014 | Accepted after revision: Sept 302014

Conflict of interest: Disclosures can be found alongside the online version of this article at err.ersjournals.com

Provenance: Publication of this peer-reviewed article was sponsored by Actelion Pharmaceuticals Ltd, Allschwil, Switzerland (principal sponsor, European Respiratory Review issue 134).

Copyright OERS 2014. ERR articles are open access and distributed under the terms of the Creative Commons Attribution Non-Commercial Licence 4.0. 
tricuspid annular plane systolic excursion (TAPSE) of $25 \mathrm{~mm}$, a tissue Doppler tricuspid annulus $\mathrm{S}$ wave of $19 \mathrm{~cm} \cdot \mathrm{s}^{-1}$ and a diameter of the inferior vena cava at $19 \mathrm{~mm}$ with preserved inspiratory collapse to $10 \mathrm{~mm}$. 8 years after diagnosis and initiation of a targeted monotherapy, this patient with IPAH was minimally symptomatic because of a preserved RV function.

Case 2 was a 20-year-old female was referred for severe exertional dyspnoea and oedema. A diagnosis of IPAH was made following a step-by-step approach following current guidelines [1]. She was in WHO functional class III with a 6-min walking distance of $340 \mathrm{~m}$. Right heart catheterisation showed mPAP of $52 \mathrm{mmHg}$, normal pulmonary capillary wedge pressure of $14 \mathrm{mmHg}$, high right atrial pressure (RAP) of $13 \mathrm{mmHg}$ and cardiac output of $3.5 \mathrm{~L} \cdot \mathrm{min}^{-1}$. She was initially treated with an ERA and acenocoumarol but improved only modestly. Episodes of clinical deteriorations required the addition of a phosphodiesterase type-5 inhibitor (PDE5i) and, eventually, continuous intravenous epoprostenol. She declined listing for lung transplantation. 7 years later, she was in WHO functional class III and walked $330 \mathrm{~m}$ in $6 \mathrm{~min}$. Her CPET showed a $V^{\prime} \mathrm{O}_{2}$ peak of $10 \mathrm{~mL} \cdot \mathrm{kg}^{-1} \cdot \mathrm{min}^{-1}$ and $V^{\prime} \mathrm{E} / V^{\prime} \mathrm{CO}_{2}$ of 58 . Right heart catheterisation showed an essentially unchanged haemodynamic profile. Doppler echocardiography showed a TRV of $4 \mathrm{~m} \cdot \mathrm{s}^{-1}$ with markedly dilated right heart chambers and a septal shift, a TAPSE of $14 \mathrm{~mm}$, a tissue Doppler imaging of tricuspid annulus $S$ wave of $8 \mathrm{~cm} \cdot \mathrm{s}^{-1}$ and a diameter of the inferior vena cava of $21 \mathrm{~mm}$ with no inspiratory collapse. There also was a pericardial effusion. 7 years after diagnosis and institution of targeted therapies, this patient with IPAH was severely symptomatic because of advanced RV failure.

These two cases represent the extremes of different evolutions of the clinical course of IPAH. The difference between the two is explained by different RV function adaptation to afterload. Case 1 has a preserved $\mathrm{RV}$ systolic function despite a mPAP approximately four times higher than the average normal of 12-15 mmHg. Case 2 has a failing right ventricle in the face of similarly increased PAP. These examples illustrate the importance of RV function as a major determinant of functional state, exercise capacity and outcome in severe pulmonary hypertension, as has been recognised in recent years [2].

\section{RV failure}

How does the right ventricle fail in pulmonary arterial hypertension $(\mathrm{PAH})$ ? The normal right ventricle is thin-walled and crescent-shaped, designed to function as a flow generator accommodating the entire systemic venous return to the heart [3]. Such a structure is vulnerable to any acute rise in wall stress. A brisk increase in PVR, for example produced by pulmonary arterial constriction to mimic massive pulmonary embolism, induces acute dilatation and rapid pump failure of the right ventricle [4]. However, a gradual increase in PVR allows for RV adaptation and remodelling, like the left ventricle facing a progressive increase in systemic vascular resistance $[5,6]$. Beat-to-beat changes in preload or afterload are accompanied by a heterometric dimension adaptation described by Starling's law of the heart. Sustained changes in load are associated with a homeometric contractility adaptation referred to as Anrep's law of the heart after the initial observation in 1912 by VON ANREP [7] of rapid increase in left ventricular (LV) contractility in response to an aortic constriction.

Homeometric adaptation to afterload (that is, without chamber dilatation) has been demonstrated in right ventricles exposed to pulmonary arterial constriction and under conditions of constant coronary perfusion [8]. Failure of systolic function, or contractility, to increase in response to loading conditions results in a heterometric adaptation allowing for maintained stroke volume (SV) at the cost of increased end-diastolic volume (EDV) $[5,6]$.

Therefore, it is possible to define RV failure as a dyspnoea fatigue syndrome with eventual systemic venous congestion, caused by the inability of the right ventricle to maintain flow output in response to metabolic demand without heterometric adaptation, and consequent increase in right heart filling pressures. This definition encompasses a spectrum of clinical situations, from preserved maximum cardiac output and aerobic exercise capacity at the price of increased RV EDVs and wall thickness (thus, raised diastolic filling pressures) to low-output states with small RV volumes at rest. This definition was endorsed at the 5th World Symposium on Pulmonary Hypertension held in 2013, in Nice, France [2].

\section{Systolic function of the right ventricle}

The homeometric adaptation is about systolic function, and has to be understood with reference to gold standard measurements of contractility. In vitro, myocardial fibre contractility is defined by an active tension-length relationship. In vivo, ventricular contractility is defined by a maximal elastance (Emax), or the maximum slope of a pressure-volume relationship measured continuously during the cardiac cycle (i.e. the "pressure-volume loop") [2, 5, 9, 10]. 
The Emax of the left ventricle coincides with end-systole and, thus, is equal to the ratio between end-systolic pressure (ESP) and end-systolic volume (ESV) defining end-systolic elastance (Ees). Left ventricular Ees is equal to Emax measured at the upper left corner of a square-shaped pressure-volume loop [11].

Because of naturally low pulmonary vascular impedance, the normal RV pressure-volume loop has a triangular rather than square shape and Emax occurs before the end of ejection or end systole [12]. However, a satisfactory definition of Emax can be obtained by the generation of a family of pressurevolume loops at decreasing venous return, e.g. generated by progressive inferior vena cava occlusion with balloon inflation [12].

Increasing impedance in pulmonary hypertension changes the shape of the RV pressure-volume loop so that Emax is closer to peak systolic pressure $[13,14]$. Examples of pressure-volume loops from two PAH patients are shown in figure 1.

Even though there has been recent progress in three-dimensional echocardiography and magnetic resonance imaging (MRI) is available in several pulmonary hypertension references centres, instantaneous pressure and volume measurements of the right ventricle are not yet possible at the bedside. Manipulations of venous return through the insertion of a vena cava balloon catheter to generate families of pressure-volume loops adds to the invasiveness of right heart catheterisation, and is ethically problematic. A Valsalva manoeuvre to generate a family of RV pressure-volume loops at decreased venous return was recently introduced and validated [14]. The practicality and clinical relevance of this approach will require confirmation.

Since Emax is insensitive to immediate changes in preload or afterload, single beat methods have been developed, initially for the left ventricle [15] but following this were adapted to the right ventricle [16]. The single-beat method relies on a maximum pressure $\left(P_{\max }\right)$ calculated from a nonlinear extrapolation of the early and late portions of a RV pressure curve, an integration of pulmonary flow or direct measurement of RV volume curve, and synchronisation of the signals. Emax is estimated from the slope of a tangent from $P \max$ to the pressure-volume curve. This is shown in figure 2.

Pmax corresponds to the pressure the right ventricle would generate during a non-ejecting beat at EDV. An excellent agreement between $P$ max measured directly, by clamping the main pulmonary artery for one beat, and calculated $P \max$ has been demonstrated in a large animal experimental preparation with no pulmonary hypertension or a mild increase in PAP induced by breathing low oxygen [16]. Whether the calculated Pmax is equally valid in patients with severe pulmonary hypertension has not been yet established with certainty.

Measurements of RV Emax with conductance catheter technology and inferior vena cava balloon obstruction have been reported in normal subjects [18]. A limited number of Emax determinations have been reported in patients with PAH either using the single-beat approach, fluid-filled catheters and MRI [19], or a multiple-beat approach with venous return decreased by a Valsalva manoeuvre and conductance catheters [14].
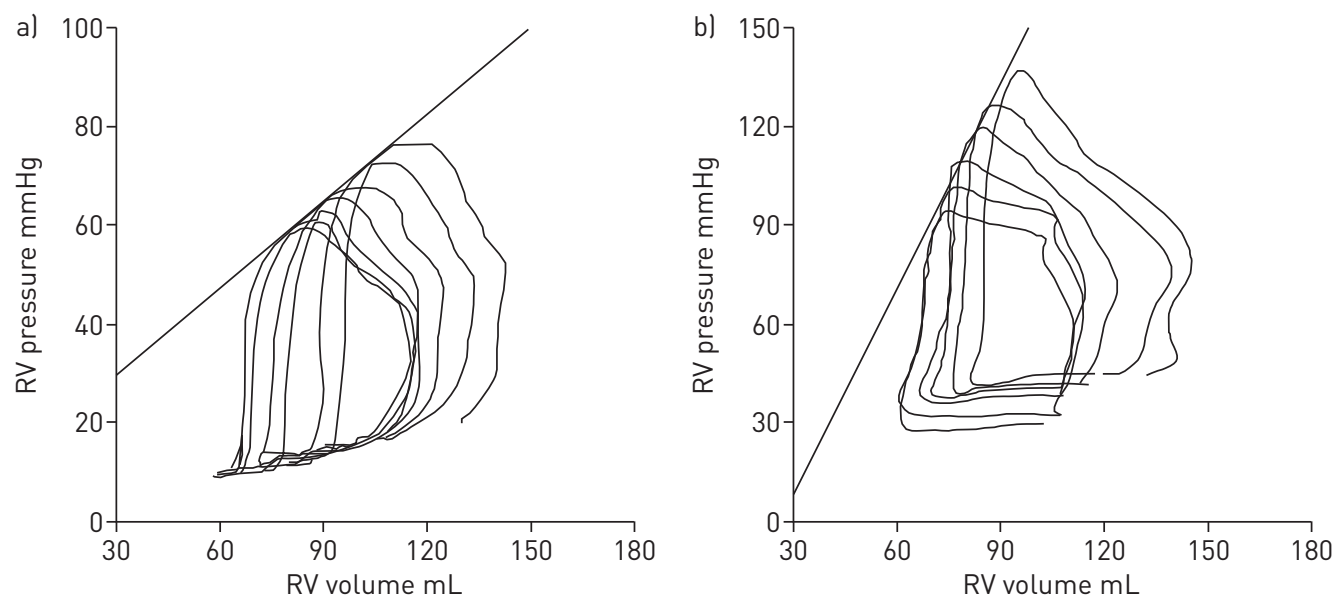

FIGURE 1 Right ventricular (RV) pressure-volume loops at decreasing venous return in a patient with a) systemic sclerosis-associated pulmonary arterial hypertension (PAH) and b) idiopathic $\mathrm{PAH}$. The mean pulmonary artery pressure of both patients was similar. The slope of linearised maximum elastance pressure-volume relationship was higher in the patient with IPAH, indicating higher contractility. Note the maximum RV pressure close to the pressure at maximum elastance in both patients. Reproduced from [14] with permission from the publisher. 

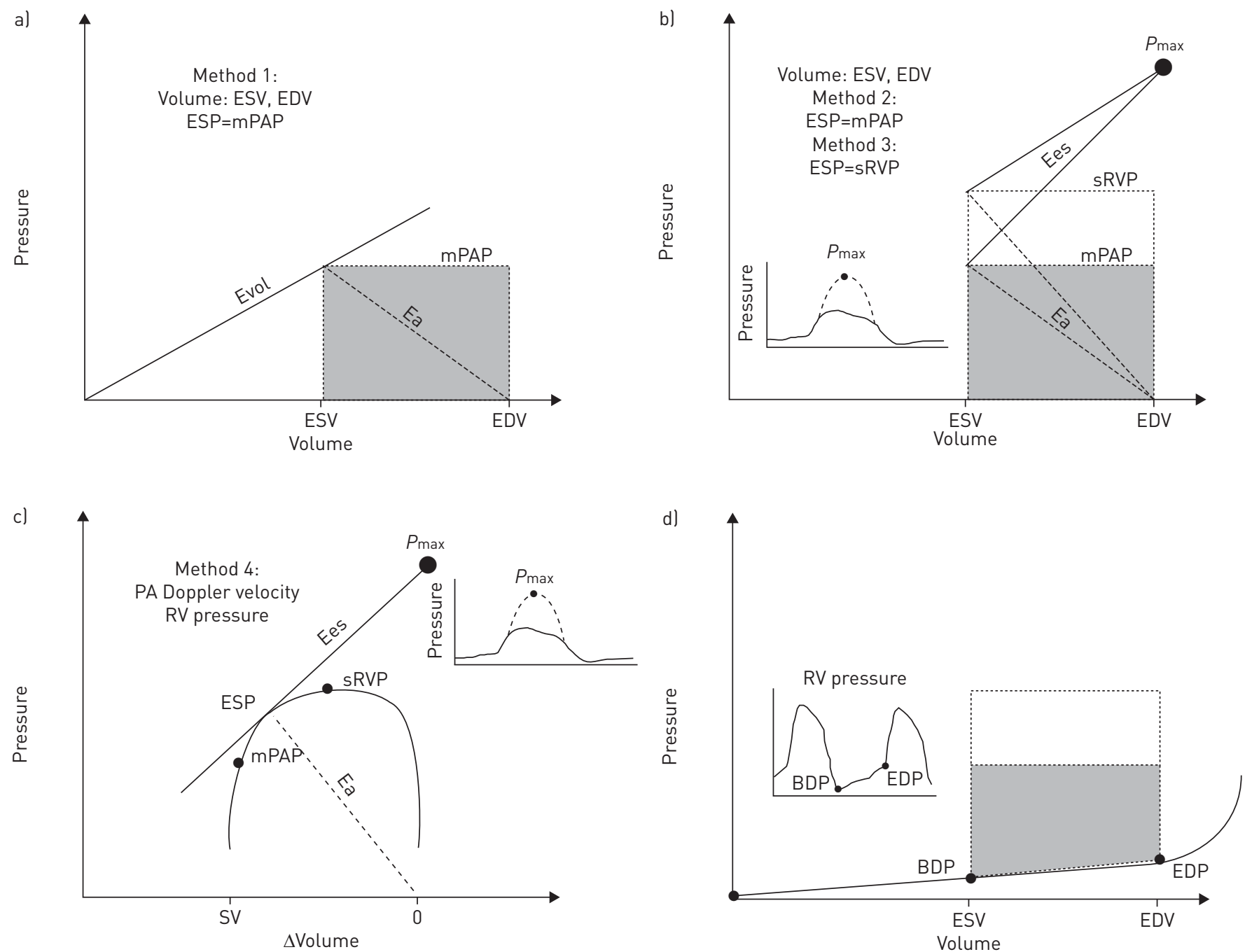

FIGURE 2 The a) volume, b) pressure, c) single-beat and d) diastolic stiffness methods used to estimate right ventricular (RV)-arterial coupling and diastolic stiffness. a, b), Arterial elastance (Ea) is calculated from the ratio of end-systolic pressure (ESP) to stroke volume (SV). a) End-systolic elastance (Ees) as an approximation of maximum elastance is estimated by the ratio of ESP to end systolic volume (ESV), which results in a simplified Ees/Ea of SV/ESV. b) Maximum pressure $\left(P_{\max }\right)$ is estimated from the non-linear extrapolation of the early systolic and diastolic portions of the RV pressure curve. Ees is then the ratio of $P \max -\mathrm{mPAP} / \mathrm{SV}$, where mPAP is mean pulmonary artery pressure. This results in a simplified Ees/Ea of Pmax/ESP-1. c) Ees is calculated as a straight line drawn from the Pmax tangent to sRVP-relative change in volume relationship. d) Diastolic stiffness $(\beta)$ is calculated by fitting the non-linear exponential, $\mathrm{P}=\alpha\left(\mathrm{e}^{\mathrm{V} \beta}-1\right)$, to the pressure and volume measured at the beginning of diastole. Where P is pressure, $\alpha$ is curve fit constant and V is volume. EDV: end diastolic volume; Evol: Ees by the volume method; sRVP: systolic RV pressure; PA: pulmonary artery; BDP: beginning diastolic pressure; EDP: end-diastolic pressure. Reproduced from [17] with permission from the publisher.

The single beat approach with high-fidelity Millar catheters and integration of a transonic measurement of pulmonary flow was reported in a patient with a systemic right ventricle in the setting of congenitally corrected transposition of the great arteries (where the right ventricle is the subaortic ventricle) [20].

Most recently, high-fidelity RV pressure and volume measurements, and single-beat Emax calculations have been reported in a small series of patients with chronic thromboembolic pulmonary hypertension (CTEPH), a condition with similar symptomatology to that of PAH [21]. This limited size report confirms the importance of systolic function adaptation with an increased Emax to maintain RV-arterial coupling in the face of severe increases in PAP, in agreement with previous studies in various animal species [10].

\section{Coupling of systolic function to afterload}

There is often confusion about the concept of "load independency" of indices of ventricular systolic function or contractility. What this really means is immediate beat-by-beat independency. Contractility, or Emax, adapts to afterload after several beats (starting after 20-30 s), with full expression of homeometric 
adaptation replacing initial heterometric adaptation within a couple of minutes, without requirement of a hypertrophic response [22]. Therefore, it is important to correct Emax for afterload.

Afterload can be measured either as maximum wall stress, integration of the forces that oppose ventricular ejection or hydraulic load and arterial elastance ( $\mathrm{Ea}$ ) [9]. Ea is defined by the ratio between pressure at Emax and SV. The interest of Ea is that it corresponds to the hydraulic load faced by the ventricle, and can be measured together with Emax on the same pressure-volume loop (fig. 2).

Thus, contractility corrected for afterload is defined by the Emax/Ea ratio. Experimental work and mathematical modelling have allowed the definition of an optimal mechanical coupling of $\mathrm{Emax} / \mathrm{Ea}=1$, but an optimal energy transfer from the ventricle to the arterial system at an Emax/Ea ratio of 1.5-2 [5].

\section{RV-arterial coupling measurements in pulmonary hypertension}

Measurements of the Emax/Ea ratio have been reported in various models of pulmonary hypertension [10]. RV coupling was preserved with increased Emax to match Ea in models of hypoxic pulmonary vasoconstriction, pulmonary embolism, pulmonary artery banding, early endotoxic shock and short-term (3 months) aortic-pulmonary shunting in piglets. However, RV-arterial coupling deteriorated with a decrease in the Emax/Ea ratio because of an insufficient adaptive increase in Emax in late endotoxic shock, monocrotaline-induced pulmonary hypertension, long-term (6 months) aortic-pulmonary shunting in piglets and mild pulmonary hypertension in over pacing-induced heart failure. Deterioration of RV-arterial coupling was constantly associated with increased EDV. Thus, there is compelling experimental evidence of predominant RV systolic function adaptation to increased afterload in pulmonary hypertension, but with RV-arterial uncoupling and increased RV volumes in the context of inflammation (endotoxaemia and monocrotaline), long-term increase in PVR or heart failure.

Measurements of both Emax and Ea have been reported in a limited number of patients with PAH. In a study of six patients with IPAH but no clinical signs of RV failure, compared to six controls, KuEHNE et al. [19] measured RV volumes and pressures with MRI and fluid-filled catheters, respectively. They synchronised the signals and calculated Emax and Ea using the single-beat method [19]. In these patients with IPAH, compared with controls, Emax was increased three-fold from $1.1 \pm 0.1$ to $2.8 \pm 0.5 \mathrm{mmHg} \cdot \mathrm{mL}^{-1}$, but Ea increased from $0.6 \pm 0.5$ to $2.7 \pm 0.2 \mathrm{mmHg} \cdot \mathrm{mL}^{-1}$, thus the Emax/Ea ratio decreased from $1.9 \pm 0.2$ to $1.1 \pm 0.1 \mathrm{mmHg} \cdot \mathrm{mL}^{-1}$. However, RV volumes were not increased, indicating "sufficient" coupling, at least in resting conditions. TEDFORD et al. [14] reported on RV-arterial coupling in five patients with IPAH and seven patients with systemic sclerosis (SSc)-associated PAH. In that study, RV volumes and pressures were measured with conductance catheters and Emax was defined by a family of pressure-volume loops as venous return decreased by a Valsalva manoeuvre (validated against inferior vena cava obstruction). In IPAH patients, Emax was $2.3 \pm 1.1 \mathrm{mmHg} \cdot \mathrm{mL}^{-1}$, Ea was $1.2 \pm 0.5 \mathrm{mmHg} \cdot \mathrm{mL}^{-1}$ and Emax/Ea was preserved at $2.1 \pm 1.0 \mathrm{mmHg} \cdot \mathrm{mL}^{-1}$. In SSc-PAH patients, Emax decreased to $0.8 \pm 0.3 \mathrm{mmHg} \cdot \mathrm{mL}^{-1}$ in the presence of Ea at $0.9 \pm 0.4 \mathrm{mmHg} \cdot \mathrm{mL}^{-1}$, thus Emax/Ea was decreased to $1.0 \pm 0.5 \mathrm{mmHg} \cdot \mathrm{mL}^{-1}$. Additionally, seven patients with SSc but without pulmonary hypertension maintained a preserved coupling (Emax/Ea $\left.2.3 \pm 1.2 \mathrm{mmHg} \cdot \mathrm{mL}^{-1}\right)$. Two examples are shown in figure 1.

Along with these studies in patients with PAH, WAUTHY et al. [20] reported a case of a systemic right ventricle in an asymptomatic young adult with a congenitally corrected transposition of the great arteries. The systemic right ventricle had an Emax of $1.26 \mathrm{mmHg} \cdot \mathrm{mL}^{-1}$, while Ea was $1.1 \mathrm{mmHg} \cdot \mathrm{mL}^{-1}$ and Emax/Ea was $1.2 \mathrm{mmHg} \cdot \mathrm{mL}^{-1}$. The pulmonary left ventricle had an Emax of $0.39 \mathrm{mmHg} \cdot \mathrm{mL}^{-1}$, while Ea was $0.23 \mathrm{mmHg} \cdot \mathrm{mL}^{-1}$ and Emax/Ea was $1.7 \mathrm{mmHg} \cdot \mathrm{mL}^{-1}$.

MCCABE et al. [21] reported Emax and Ea measurements in 10 patients with CTEPH. Pressures and volumes were measured with a conductance catheter. The results were compared with those of seven patients with thromboembolic pulmonary vascular disease but no pulmonary hypertension and seven normal controls. In the CTEPH patients, Emax was $1.1 \pm 0.4 \mathrm{mmHg} \cdot \mathrm{mL}^{-1}$, Ea $1.9 \pm 0.7 \mathrm{mmHg} \cdot \mathrm{mL}^{-1}$ and Emax/Ea was $0.6 \pm 0.1 \mathrm{mmHg} \cdot \mathrm{mL}^{-1}$. In the thromboembolic pulmonary vascular disease patients, Emax was $0.6 \pm 0.3 \mathrm{mmHg} \cdot \mathrm{mL}^{-1}$, Ea was $0.5 \pm 0.2 \mathrm{mmHg} \cdot \mathrm{mL}^{-1}$ and Emax/Ea was $1.3 \pm 0.4 \mathrm{mmHg} \cdot \mathrm{mL}^{-1}$. In the controls, Emax was $0.4 \pm 0.2 \mathrm{mmHg} \cdot \mathrm{mL}^{-1}$, Ea was $0.3 \pm 0.1 \mathrm{mmHg} \cdot \mathrm{mL}^{-1}$ and Emax/Ea was $1.5 \pm 0.3 \mathrm{mmHg} \cdot \mathrm{mL}^{-1}$.

Altogether, these results confirm the predominant role of homeometric adaptation of the right ventricle to increased afterload. However, RV-arterial uncoupling occurs when the hydraulic load remains too high for too long, or in the presence of systemic disease. On the methodological side, it is apparent that Emax and Ea measurements show variability, with a trend toward higher values when measurements are based on families of pressure-volume loops rather than on the single-beat method. Furthermore, RV volumes measured using a conductance catheter appear to underestimate ESV and EDV compared with MRI measurements. Targeted therapies in PAH patients might also have affected these results. 


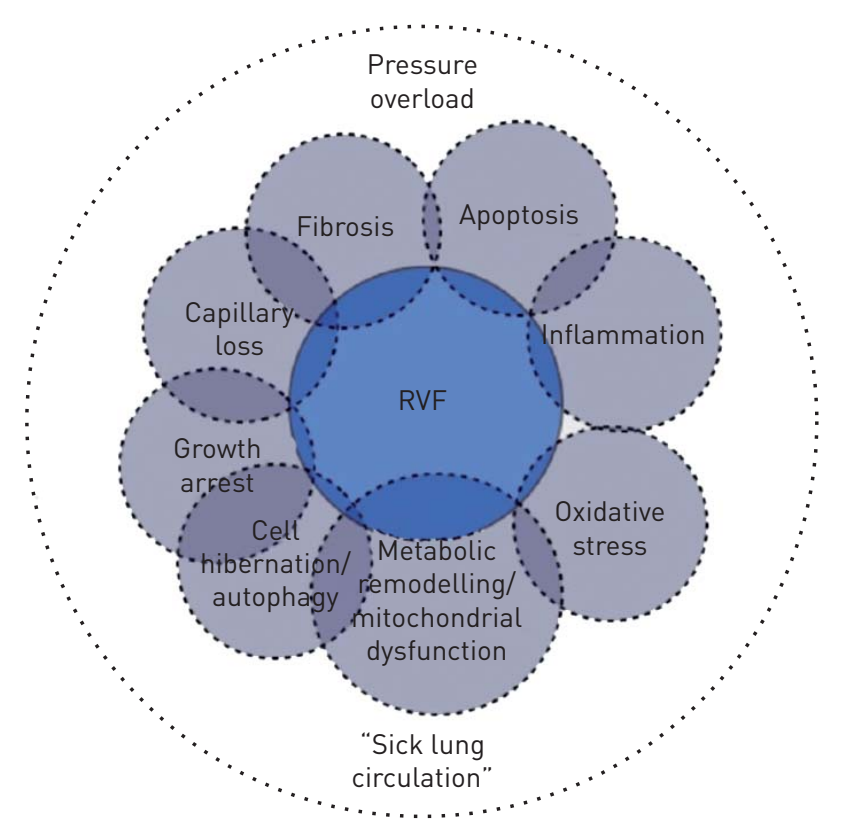

FIGURE 3 The right ventricle between "a rock and a hard place", challenged by the pressure overload and the sick lung circulation. The grey circles symbolise cellular and molecular mechanisms. RVF: right ventricular failure. Reproduced from [25] with permission from the publisher.

\section{Pathobiology of RV-arterial coupling}

The current understanding of the pathophysiology of RV failure involves neurohumoral activation, expression of inflammatory mediators, apoptosis, capillary loss, oxidative stress and metabolic shifts, with variable fibrosis and hypertrophy (fig. 3) [2, 23-25].

RV ischaemia may play a role in later stages [26]. The exact sequence of events and interactions is being explored, and each still has to be referred to sound measurements of function, as illustrated in recent studies that showed inflammation and apoptosis correlated to decreased Emax/Ea in acute [27], as well as chronic [28], models of RV failure as a universal mechanism.

\section{Pharmacology of RV-arterial coupling in pulmonary hypertension}

There are no reported data on the effects of drugs on RV-arterial coupling in PAH. Therefore, one has to rely on translations from experimental animal data, with all possible caveats inherent to this approach.

Catecholamines are, in principle, not recommended in severe pulmonary hypertension because of their potential to induce pulmonary vasoconstriction, excessive tachycardia and arrhythmia [29]. Moreover, catecholamines have been associated with increased mortality in RV failure [30]. The latter effect is probably due to the fact that these drugs are prescribed in the most severely ill patients, but the data nevertheless cause concern. Experimental animal data offer a less negative view. Low-dose dobutamine increased RVarterial coupling by an inotropic effect with a slight decrease in PVR [31]. Low-dose noradrenaline improved RV-arterial coupling through an exclusive positive inotropic effect, which was, however, less pronounced than with low-dose dobutamine [31]. Low-dose catecholamines are often used for short periods of time in the intensive care setting to stabilise patients with acute or acute-on-chronic RV failure, buying time for fluid management and treatments targeting the pulmonary circulation. However, no recommendation can be made as this treatment is based on pathophysiological rationale and clinical experience; it is not evidence-based.

Quite opposite is the idea that $\beta$-blockers might improve RV failure, as has been shown to be effective and is actually recommended in LV failure. Heart failure, whether global or limited to the right ventricle, is associated with progressive worsening, at least in part, attributable to excessive neurohumoral activation [25]. Experimental animal evidence is contrasting. Acute administration of propranolol reduced RV-arterial coupling through combined negative inotropy and pulmonary vasoconstriction during acute hypoxic exposure [16]. However, chronic administration of bisoprolol improved RV-arterial coupling by an improved contractility in monocrotaline-induced pulmonary hypertension [32]. Chronic administration of carvedilol or metoprolol improved echocardiography of RV function in pulmonary hypertension induced by the 
vascular endothelial growth factor receptor antagonist SU5416 combined with hypoxia or monocrotaline injection [33]. Accordingly, two randomised controlled trials of low-dose $\beta$-blockers have been initiated in patients with PAH. Results of these trials are not yet available.

There have been suggestions that drugs targeting the pulmonary circulation in PAH might also have intrinsic beneficial effects on the right ventricle [34, 35]. However, the experimental evidence for such direct myocardial effects in intact animal models of pulmonary hypertension is scarce. Acute administration of epoprostenol or inhaled nitric oxide improved RV-arterial coupling through exclusive pulmonary vasodilation effects in over circulation-induced pulmonary hypertension [36]. Acute epoprostenol partially restored RV-arterial coupling through an exclusive pulmonary vascular effect in pulmonary bandinginduced persistent RV failure [37], or was associated with maintained RV-arterial coupling because of decreased contractility in proportion to decreased PVR in hypoxia [38]. Sildenafil improved RV-arterial coupling in hypoxia because of exclusive pulmonary vasodilation effects [39], but improved the coupling by a positive inotropic effect in monocrotaline-induced pulmonary hypertension [40]. Bosentan had no intrinsic effect on contractility in pulmonary hypertension after 3 months of aortic-pulmonary shunting [41]. Thus, at the present stage, it is safe to state that there is no convincing evidence that classes of drugs shown effective in the treatment of $\mathrm{PAH}$ have positive inotropic effects.

\section{Simplified methods for the measurement of RV-arterial coupling} Volume measurements

The Emax/Ea ratio presents a common pressure term and, thus, can be simplified to a ratio of volumes:

\section{(ESP/ESV)/(ESP/SV) becomes SV/ESV}

SANZ et al. [42] measured ESV and SV by MRI and showed that the SV/ESV ratio is initially preserved in patients with mild pulmonary hypertension but decreases with increasing disease severity. A problem with the SV/ESV ratio is the inherent assumption that the ESP-ESV relationship is linear and crosses the origin [43]. This is incorrect, because ventricular volume at a zero filling pressure has to be positive. Therefore, the ESP-ESV relationship underestimates Emax (fig. 2). Another problem is that the SV/ESV ratio assumes that Ees coincides with Emax. As already discussed, this assumption is also incorrect (figs 1 and 2).

It can ideally be reasoned that the SV/ESV ratio includes the information of RV ejection fraction (RVEF) or SV/EDV in a less preload-dependent manner, but the relevance of this remains to be established. The SV/ ESV ratio as a simple volume measurement of RV-arterial coupling requires further evaluation and estimation of functional and prognostic relevance. Most recently, VANDERPOOL et al. [17] reported on the prognostic value of the SV/ESV ratio as a predictor of survival in a relatively small cohort of patients referred for severe pulmonary hypertension. In that study, a multivariable analysis showed the SV/ESV ratio, not ejection fraction, to be an independent predictor of outcome [17].

VAN DE VEERDONK et al. [44] recently reported on the negative impact on outcome of decreased RVEF despite a targeted therapy-associated decreased PVR in patients with PAH. This study highlighted the importance of RV function in the prognostication of PAH [45]. However, vasodilation therapies may be a confounding factor in the interpretation of RVEF. Systemic vasodilation effects of targeted therapies in PAH may increase systemic venous return and increase EDV, which decreases ejection fraction if SV remains essentially unchanged, while increased cardiac output may decrease PVR without any change in the functional state of pulmonary circulation [45].

Advances in three-dimensional echocardiography now offer the prospect of easier bedside measurements of RV volumes [46], and thus of ejection fraction or SV/ESV for the evaluation of RV-arterial coupling.

\section{Pressure measurements}

Another simplified approach for the measurement of RV-arterial coupling, introduced by TRIP et al. [43], relies on $P \max$ calculated from a RV pressure curve (easily obtained during right heart catheterisation), mPAP taken as a surrogate of ESP and RV volume measurements by MRI. The authors calculated Emax as $(P \max -\mathrm{mPAP}) /(\mathrm{EDV}-\mathrm{ESV})$, which can be simplified as mPAP/ESV assuming $\mathrm{V}_{0}=0 . \mathrm{V}_{0}$ is the extrapolated volume intercept of the linear best fit of a multipoint maximum elastance pressure-volume relationship. According to this "pressure method", Emax/Ea $=P_{\max } / \mathrm{mPAP}-1$ (fig. 2).

The results showed that mPAP/ESV was lower than $(P \max -\mathrm{mPAP}) / \mathrm{SV}$, on average about half the value, while $\mathrm{V}_{0}$ ranged from -8 to $171 \mathrm{~mL}$ and was correlated to EDV and ESV. From this the authors concluded that $\mathrm{V}_{0}$ is dependent on RV dilatation, and thus the estimated Emax is more preload-dependent than previously assumed [43]. This may be a matter of debate. In the meantime, VANDERPOOL et al. [17] showed that Emax/Ea estimated by the pressure method did not emerge as an independent predictor of survival. 


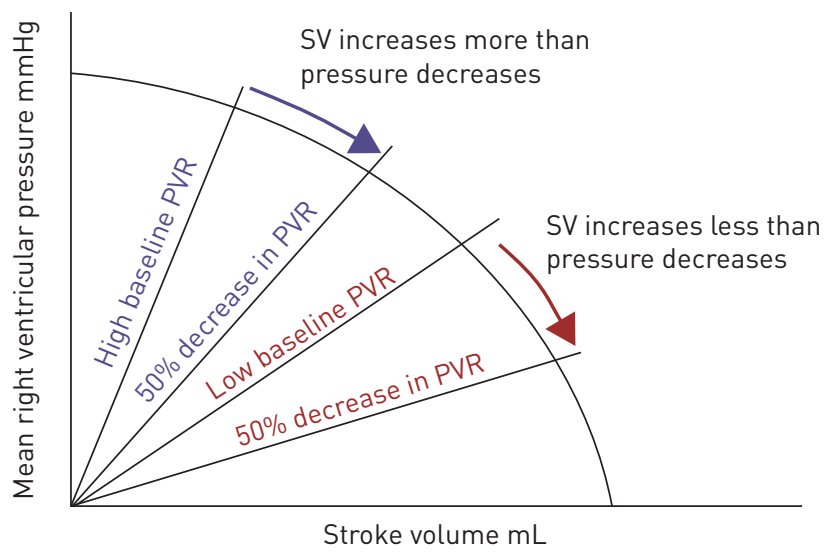

FIGURE 4 A pump function graph demonstrating mean right ventricular pressure as a function of stroke volume (SV). Maximum mean right ventricular pressure is calculated from nonlinear extrapolations of early and late systolic portions of the right ventricular pressure curve. SV is calculated by dividing cardiac output by heart rate. The curve is a parabolic fit of two data points: maximum mean right ventricular pressure and measured mean right ventricular pressure/SV. At high right ventricular pressure, a small decrease in pressure results in a large increase in SV. PVR: pulmonary vascular resistance. Reproduced from [2] with permission from the publisher.

\section{Alternative methods to evaluate RV-arterial coupling \\ The pump function graph}

The coupling of RV function to the pulmonary circulation can also be described by pump function curves relating mean ventricular pressure to SV, as shown by ELZINGA and WESTERHOF [47] for the left ventricle. A pump function graph is built from measurements of mean RV pressure and SV, a Pmax calculated at zero SV and a parabolic extrapolation to a zero pressure SV [2,9]. In this representation, an increase in preload shifts the curve to greater SV with no change in shape, while an increased contractility leads to a higher $P \max$ with no change in maximum SV. The pump function graph helps to understand that at high RV pressures, a slight decrease in pressure markedly increases SV while at low RV pressures a marked fall in pressure has minimal effect on SV (fig. 4) [2].

The pump function graph has been used to demonstrate more severe RV failure at any given level of mPAP in SSc-PAH compared with IPAH [48].

The limitations of the pump function graph are in its sensitivity to changes in preload and, as already mentioned, to the use of mean RV pressure or mPAP as surrogates of maximum elastance RV pressure.

\section{The contractile reserve}

Systolic function adaptation to afterload can also be tested dynamically to determine a contractile reserve, or the capacity to increase contractility at a given level of loading. Contractile or ventricular reserve determined using exercise or pharmacological stress tests (typically an infusion of dobutamine) has been shown to be a strong predictor of outcome in heart failure [49]. The evaluation of RV contractile reserve has not been reported in patients with pulmonary hypertension until now. In rats with pulmonary arterial banding, Emax was shown to increase to the same extent in response to an infusion of dobutamine at a dose of $2.5 \mu \mathrm{g} \cdot \mathrm{kg}^{-1} \cdot \mathrm{min}^{-1}$ compared with controls, suggesting preserved systolic function in this pulmonary hypertension model [50].

A simple noninvasive approach was recently introduced by GRüNIG et al. [51]. In that study, Doppler echocardiography was used to measure RV systolic pressure from the maximum TRV at rest and exercise in 124 patients with either PAH or CTEPH. An exercise-induced increase $>30 \mathrm{mmHg}$ was a strong predictor of exercise capacity and survival, the inference being that if RV contractile reserve remains good, then a pressure response to exercise can be successfully mounted despite increased afterload. Further studies will explore improved indices with incorporation of volume measurements and ESP determinations, as this is now becoming possible using noninvasive bedside methodology.

\section{Surrogate measurements of RV-arterial coupling}

RV systolic function can be estimated by a series of invasive and noninvasive measurements that are commonly available in daily clinical practice. 
Right heart catheterisation allows for measurements of PAP, RAP and cardiac output (Fick or thermodilution) and thus calculations of RV function curves such as cardiac output, SV or stroke work. Stroke work calculated as mPAP $\times$ SV ignores the pulsatile component of work. It has recently been estimated that the pulsatile component of stroke work amounts to $23 \%$ of total work independent of type and severity of pulmonary hypertension, so that total stroke work is $1.3 \mathrm{mPAP} \times \mathrm{SV}$ [52]. This fixed relationship is explained by the constancy of the resistance-compliance time constant of the pulmonary circulation, or PVR $\times$ pulmonary arterial compliance [2]. However, routine right heart catheterisation with fluid-filled thermodilution catheters is limited because it cannot measure ventricular volumes.

This limitation of right heart catheterisation is overcome by MRI or, more recently, three-dimensional echocardiography. The limitation of imaging is in the absence of direct pressure measurements.

A series of imaging-derived indices of RV systolic function have been proposed. The most robust appears to be ejection fraction as determined by MRI [2]. However, MRI is not generally available and is impossible to integrate in bedside clinical evaluations in daily life. Doppler echocardiography is more flexible, especially since portable devices have been developed allowing easy integration of measurements during the clinical examination. Doppler echocardiography measurements of RV function shown to be of functional and/or prognostic relevance include: the fractional area change (measure on apical four-chamber view); TAPSE (preferably measured in M-mode) tissue Doppler imaging of the tricuspid annulus systolic velocity $\mathrm{S}$ wave; and isovolumic acceleration or maximum velocity and strain or strain rate $[53,54]$. Isovolumic phase indices such as the isovolumic acceleration or maximum velocity are probably less preload dependent, and as such the closest estimates of Emax measurements $[55,56]$.

\section{Diastolic function}

Even though systolic function adaptation to afterload is central to the occurrence of RV failure symptomatology, diastolic function changes must also be taken into consideration.

Diastolic function is described by a diastolic elastance curve determined by a family of pressure-volume loops at variable loading. It is curvilinear, thus impossible to summarise as a single number. Several formulas have been proposed [9]. Most recently, RAIN et al. [57] reported 21 patients with PAH in whom RV diastolic stiffness was estimated by fitting a nonlinear exponential curve through the diastolic pressurevolume relationships, with the formula $\mathrm{P}=\alpha\left(\mathrm{e}^{\mathrm{V} \beta}-1\right)$, where $\alpha$ is a curve fitting constant and $\beta$ a diastolic stiffness constant (fig. 2). In that study, the diastolic stiffness constant $\beta$ was closely associated with disease severity. The pathogenesis of RV diastolic dysfunction was related to increased RV collagen content (i.e. fibrosis) and stiffness of the RV sarcomeres that, in turn, was due to reduced phosphorylation of titin, a key protein regulating myocyte stiffness. Similarly calculated $\beta$ in a recent clinical study [17] showed that it was not an independent predictor of outcome, but this requires confirmation.

A series of surrogate measurements of diastolic function are provided by Doppler echocardiography, these include: 1) isovolumic relaxation time and a decreased ratio of transmittal $\mathrm{E}$ and A waves or mitral annulus tissue Doppler imaging E'/A' waves; 2) increased right atrial or RV surface areas on apical four-chamber views; 3 ) altered eccentricity index on a parasternal short axis view; 4) estimates of RAP from RV diastolic function indices or inferior vena cava dimensions; 5) pericardial effusion; and 6) the so-called Tei index, which is the ratio of isovolumic time intervals to ventricular ejection time, and thus integrates diastolic and systolic function $[53,54]$.

\section{Ventricular interaction}

RV function also has to be understood in the context of its direct and indirect interactions with LV function. Direct interaction, or ventricular interdependence, is defined as the forces that are transmitted from one ventricle to the other through the myocardium and pericardium, independent of neural, humoral or circulatory effects [58]. Diastolic ventricular interaction refers to the competition for space within the nondistensible pericardium when the right ventricle dilates, which alters LV filling and may be a cause of inadequate cardiac output response to metabolic demand. Right heart catheterisation and imaging studies have shown that in patients with severe pulmonary hypertension, MPAP and LV peak filling rate are altered in proportion to decreased RV ejection fraction [59]. Systolic interaction refers to positive interaction between RV and LV contractions. It can be shown experimentally that aortic constriction, and enhanced LV contraction, markedly improves RV function in animals with pulmonary arterial banding [60]. Similarly, in electrically isolated ventricular preparations in the otherwise intact dog heart, LV contraction contributes a significant amount $(\sim 30 \%)$ to both RV contraction and pulmonary flow [61]. This is explained by a mechanical entrainment effect, but also by LV systolic function determining systemic blood pressure which is an essential determinant of RV coronary perfusion. Increased RV filling pressures and excessive decrease in blood pressure may be a cause of RV ischaemia and decreased contractility. 
Treatment of acute afterload-induced RV failure

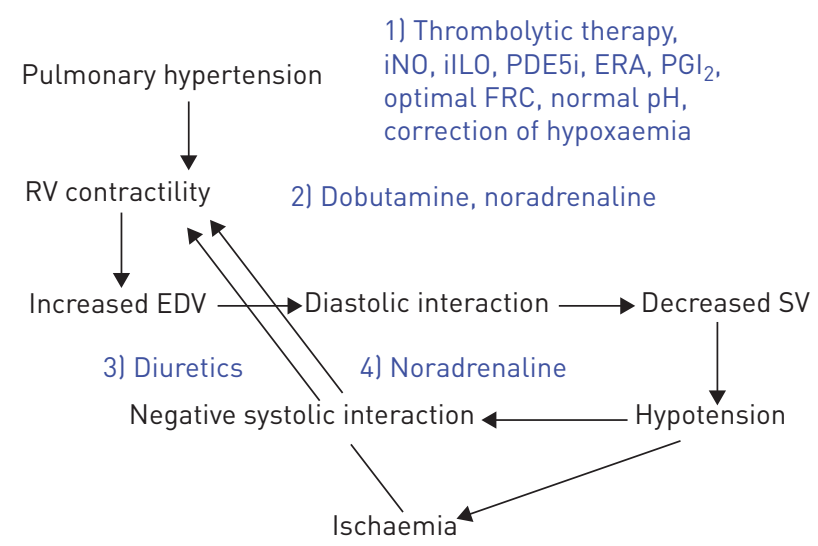

FIGURE 5 Pathophysiology of right ventricular (RV) failure. Pulmonary hypertension increases RV afterload requiring a homeometric adaptation, i.e. an increased RV contractility. When this adaptation fails, the RV enlarges with increased end-diastolic volume (EDV), decreasing left ventricle preloading because of competition for space within the pericardium. This decreases stroke volume (SV) and blood pressure, with negative systolic interaction as a cause of further RV-arterial uncoupling, which may be aggravated by RV ischaemia from decreased coronary perfusion pressure (gradient between diastolic blood pressure and right atrial pressure). Obvious targets for interventions are indicated as 1 to 4. See the A global view on RV failure section for further details. iNO: inhaled nitric oxide; iILO: inhaled iloprost; PDE5i: phosphodiesterase type-5 inhibitor; ERA: endothelin receptor antagonist; $\mathrm{PGI}_{2}$ : prostaglandin $\mathrm{I}_{2}$; FRC: functional residual capacity.

An additional cause of negative ventricular interaction highlighted by imaging studies is regional dyssynchrony and inter-ventricular asynchrony with post-systolic contraction or "shortening", which has been shown to develop in parallel with increased PAP and contributes to altered RV systolic function and LV under-filling [62]. Inter-ventricular asynchrony can be assessed by tissue Doppler imaging [63]. Right ventricular dyssynchrony can now accurately be identified and quantified by echocardiography using threedimensional speckle tracking [64].

\section{A global view on RV failure}

A simplified but clinically useful global view of RV failure is shown in figure 5. Thus, pulmonary hypertension increases RV afterload, requiring a homeometric adaptation. When this adaptation fails, the right ventricle enlarges, decreasing LV preloading because of competition for space within the pericardium. This decreases SV and blood pressure, with negative systolic interaction as a cause of further RV-arterial uncoupling, which may be aggravated by RV ischaemia from decreased coronary perfusion pressure (gradient between diastolic blood pressure and RAP).

As shown in figure 5, these interactions may allow one to identify targets of interventions. Target 1 is increased PVR, which can be controlled by: thrombolytics in severe pulmonary embolism; inhaled nitric oxide; inhaled iloprost (in the case of acute pulmonary vasoconstriction); prostacyclins; i.v. epoprostenol; and PDE5i and ERAs. Correction of decreased or increased functional residual capacity, $\mathrm{pH}$ and hypoxaemia are all causes of increased PVR in lung diseases. Target 2 is about relative insufficiency of RV contractility, which can be temporarily corrected by i.v. dobutamine. Target 3 is correction of excessive RV preload and associated negative diastolic interaction by high-dose i.v. diuretics and fluid restriction. Target 4 is correction of excessive systemic hypotension and associated negative systolic interaction and eventual $\mathrm{RV}$ ischaemia by i.v. noradrenaline.

\section{Conclusion}

The awareness of the importance of the right ventricle in PAH has increased considerably. Nowadays, there is a clearer view of the right ventricle and the pulmonary circulation as a functional unit, and imaging is being used with improved focus on functional relevance. Measurements of RV-arterial coupling allow for the identification of pulmonary vascular versus RV myocardial effects of therapeutic interventions, and could serve as useful tools for bedside translation of cell and molecular biological discoveries.

A lot of work remains to be done to identify the most relevant measurements of RV function and RVarterial coupling and their sensitivity to pharmacological interventions. Easy to implement imaging at the bedside and in outpatient clinics is becoming available in many centres, offering fascinating perspectives of renewed clinical research on right heart failure. 


\section{References}

Hoeper MM, Bogaard HJ, Condliffe R, et al. Definitions and diagnosis of pulmonary hypertension. J Am Coll Cardiol 2013; 62: Suppl. 25, D45-D50.

2 Vonk-Noordegraaf A, Haddad F, Chin KM, et al. Right heart adaptation to pulmonary arterial hypertension: physiology and pathobiology. J Am Coll Cardiol 2013; 62: Suppl. 25, D22-D33.

3 Haddad F, Hunt SA, Rosenthal DN, et al. Right ventricular function in cardiovascular disease, part I. Anatomy, physiology, aging and functional assessment of the right ventricle. Circulation 2008; 117: 1436-1448.

4 Guyton AC, Lindsey AW, Gilluly JJ. The limits of right ventricular compensation following acute increase in pulmonary circulatory resistance. Circ Res 1954; 2: 326-332.

5 Sagawa K, Maughan L, Suga H, eds, et al. Cardiac Contraction and the Pressure-Volume Relationship. Oxford, Oxford University Press, 1988.

6 Sarnoff SJ, Mitchell JH, Gilmore JP, et al. Homeometric autoregulation of the heart. Circ Res 1960; 8: 1077-1091.

7 von Anrep G. On the part played by the suprarenals in the normal vascular reactions of the body. J Physiol 1912; 45: 307-317.

8 Taquini AC, Fermoso JD, Aramendia P. Behaviour of the right ventricle following acute constriction of the pulmonary artery. Circ Res 1960: 315-318.

9 Vonk-Noordegraaf A, Westerhof N. Describing right ventricular function. Eur Respir J 2013; 41: 1419-1423.

10 Naeije R, Brimioulle S, Dewachter C. Biomechanics of the right ventricle. Pulm Circ 2014; 4: 395-406.

11 Suga H, Sagawa K, Shoukas AA. Load independence of the instantaneous pressure-volume ratio of the canine left ventricle and effects of epinephrine and heart rate on the ratio. Circ Res 1973; 32: 314-322.

12 Maughan WL, Shoukas AA, Sagawa K, et al. Instantaneous pressure-volume relationship of the canine right ventricle. Circ Res 1979; 44: 309-315.

13 Redington AN, Rigby RL, Shinebourne EA, et al. Changes in pressure-volume relation of the right ventricle when its loading conditions are modified. Br Heart J 1990; 63: 45-49.

14 Tedford RJ, Mudd JO, Girgis RE, et al. Right ventricular dysfunction in systemic sclerosis associated pulmonary arterial hypertension. Circulation Heart Fail 2013; 6: 953-963.

15 Sunagawa K, Yamada A, Senda Y, et al. Estimation of the hydromotive source pressure from ejecting beats of the left ventricle. IEEE Trans Biomed Eng 1980; 57: 299-305.

16 Brimioulle S, Wauthy P, Ewalenko P, et al. Single-beat estimation of right ventricular end-systolic pressure-volume relationship. Am J Physiol Heart Circ Physiol 2003; 284: H1625-H1630.

17 Vanderpool RR, Pinsky MR, Naeije R, et al. RV-pulmonary arterial coupling predicts outcome in patients referred for pulmonary hypertension. Heart 2014 [In press DOI: 10.1136/heartjnl-2014-306142].

18 Dell'Italia LJ, Walsh RA. Application of a time-varying elastance model to right ventricular performance in man. Cardiovasc Res 1988; 22: 864-874.

19 Kuehne T, Yilmaz S, Steendijk P, et al. Magnetic resonance imaging analysis of right ventricular pressure-volume loops: in vivo validation and clinical application in patients with pulmonary hypertension. Circulation 2004; 110 : 2010-2016.

20 Wauthy P, Naeije R, Brimioulle S. Left and right ventriculo-arterial coupling in a patient with congenitally corrected transposition. Cardiol Young 2005; 15: 647-649.

21 McCabe C, White PA, Hoole SP, et al. Right ventricular dysfunction in chronic thromboembolic obstruction of the pulmonary artery. J Appl Physiol 2013; 116: 355-363.

22 Rosenblueth A, Alanis J, Lopez E, et al. The adaptation of ventricular muscle to different circulatory conditions. Arch Int Physiol Biochim 1959; 67: 358-373.

23 Bogaard HJ, Abe K, Vonk Noordegraaf A, et al. The right ventricle under pressure. Cellular and molecular mechanisms of right heart failure in pulmonary hypertension. Chest 2009; 135: 794-780.

24 Voelkel NF, Gomez-Arroyo J, Abbate A, et al. Pathobiology of pulmonary arterial hypertension and right ventricular failure. Eur Respir J 2012; 40: 1555-1565.

25 Voelkel NF, Gomez-Arroyo J, Abbate A, et al. Mechanisms of right heart failure - a work in progress and plea for further prevention. Pulm Circ 2013; 3: 137-143.

26 Gómez A, Bialostozky D, Zajarias A, et al. Right ventricular ischemia in patients with primary pulmonary hypertension. J Am Coll Cardiol 2001; 38: 1137-1141.

27 Dewachter C, Dewachter L, Rondelet B, et al. Activation of apoptotic pathways in experimental acute afterloadinduced right ventricular failure. Crit Care Med 2010; 38: 1405-1413.

28 Belhaj A, Dewachter L, Kerbaul F, et al. Heme oxygenase-1 and inflammation in experimental right ventricular failure on prolonged overcirculation-induced pulmonary hypertension. PLoS One 2013; 8: e69470.

29 Hoeper MM, Granton J. Intensive care unit management of patients with severe pulmonary hypertension and right heart failure. Am J Respir Crit Care Med 2011; 184: 1114-1124.

30 Sztrymf B, Günther S, Artaud-Macari E, et al. Left ventricular ejection time in acute heart failure complicating precapillary pulmonary hypertension. Chest 2013; 144: 1512-1520.

31 Kerbaul F, Rondelet B, Motte S, et al. Effects of norepinephrine and dobutamine on pressure load-induced right ventricular failure. Crit Care Med 2004; 32: 1035-1040.

32 de Man FS, Handoko ML, van Ballegoij JJ, et al. Bisoprolol delays progression towards right heart failure in experimental pulmonary hypertension. Circ Heart Fail 2012; 5: 97-105.

33 Bogaard HJ, Natarajan R, Mizuno S, et al. Adrenergic blockade reverses right heart remodeling and dysfunction in pulmonary hypertensivre rats. Am J Respir Crit Care Med 2010; 182: 652-656.

34 Rich S, McLaughlin VV. The effects of chronic prostacyclin therapy on cardiac output and symptoms in primary pulmonary hypertension. J Am Coll Cardiol 1999; 34: 1184-1187.

35 Nagendran J, Archer SL, Soliman D, et al. Phosphodiesterase type 5 is highly expressed in the hypertrophied human right ventricle, and acute inhibition of phosphodiesterase type 5 improves contractility. Circulation 2007; 116: 238-248.

36 Wauthy P, Kafi AS, Mooi W, et al. Effects of nitric oxide and prostacyclin in an over-circulation model of pulmonary hypertension. J Thorac Cardiovasc Surg 2003; 125: 1430-1437. 
conjunction with pulmonary hypertension. Am J Respir Crit Care Med 2007; 175: 846-850.

38 Rex S, Missant C, Segers P, et al. Epoprostenol treatment of acute pulmonary hypertension paradoxical decrease in right ventricular contractility. Intens Care Med 2008; 34: 179-189.

39 Fesler P, Pagnamenta A, Rondelet B, et al. Effects of sildenafil on hypoxic pulmonary vascular function in dogs. J Appl Physiol 2006; 101: 1085-1090.

40 Borgdorff MA, Bartelds B, Dickinson MG, et al. Sildenafil enhances systolic adaptation, but does not prevent diastolic dysfunction, in the pressure-loaded right ventricle. Eur J Heart Fail 2012; 14: 1067-1074.

41 Rondelet B, Kerbaul F, Motte S, et al. Bosentan for the prevention of overcirculation-induced pulmonary hypertension. Circulation 2003; 107: 1329-1335.

42 Sanz J, García-Alvarez A, Fernández-Friera L, et al. Right ventriculo-arterial coupling in pulmonary hypertension: a magnetic resonance study. Heart 2012; 98: 238-243.

43 Trip P, Kind T, van de Veerdonk MC, et al. Accurate assessment of load-independent right ventricular systolic function in patients with pulmonary hypertension. J Heart Lung Transplant 2013; 32: 50-55.

44 van de Veerdonk MC, Kind T, Marcus JT, et al. Progressive right ventricular dysfunction in patients with pulmonary arterial hypertension responding to therapy. J Am Coll Cardiol 2011; 58: 2511-2519.

45 Sniderman AD, Fitchett DH. Vasodilators and pulmonary arterial hypertension: the paradox of therapeutic success and clinical failure. Int J Cardiol 1988; 20: 173-181.

46 Zhang QB, Sun JP, Gao RF, et al. Feasibility of single-beat full volume capture real-time three-dimensional echocardiography for quantification of right ventricular volume: validation by cardiac magnetic resonance imaging. Int J Cardiol 2013; 168: 3991-3995.

47 Elzinga $\mathrm{G}$, Westerhof $\mathrm{N}$. The effect of an increase in inotropic state and end-diastolic volume on the pumping ability of the feline left heart. Circ Res 1978; 42: 620-628.

48 Overbeek MJ, Lankhaar JW, Westerhof N, et al. Right ventricular contractility in systemic sclerosis-associated and idiopathic pulmonary arterial hypertension. Eur Respir J 2008; 31: 1160-1166.

49 Haddad F, Vrtovec B, Ashley EA, et al. The concept of ventricular reserve in heart failure and pulmonary hypertension: an old metric that brings us one step closer in our quest for prediction. Curr Opin Cardiol 2011; 26: $123-131$.

50 Faber MJ, Dalinghaus M, Lankhuizen IM, et al. Right and left ventricular function after chronic pulmonary artery banding in rats assessed with biventricular pressure-volume loops. Am J Physiol Heart Circ Physiol 2006; 291: H1580-H1586.

51 Grünig E, Tiede H, Enyimayew EO, et al. Assessment and prognostic relevance of right ventricular contractile reserve in patients with pulmonary arterial hypertension. Circulation 2013; 128: 2005-2015.

52 Saouti N, Westerhof N, Helderman F, et al. Right ventricular oscillatory power is a constant fraction of total power irrespective of pulmonary artery pressure. Am J Respir Crit Care Med 2010; 182: 1315-1320.

53 Bossone E, D’Andrea A, D’Alto M, et al. Echocardiography in pulmonary arterial hypertension: from diagnosis to prognosis. J Am Soc Echocardiogr 2013; 26: 1-14.

54 Roberts JD, Forfia PR. Diagnosis and assessment of pulmonary vascular disease by Doppler echocardiography. Pulm Circ 2011; 1: 160-181.

55 Ernande L, Cottin V, Leroux PY, et al. Right isovolumic contraction velocity predicts survival in pulmonary hypertension. J Am Soc Echocardiogr 2013; 26: 297-306.

56 Vogel M, Schmidt MR, Christiansen SB, et al. Validation of myocardial acceleration during isovolumic contraction as a novel non-invasive index of right ventricular contractility. Circulation 2002; 105: 1693-1699.

57 Rain S, Handoko ML, Trip P, et al. Right ventricular diastolic impairment in patients with pulmonary arterial hypertension. Circulation 2013; 128: 2016-2025.

58 Santamore WP, Dell'Italia LJ. Ventricular interdependence: significant left ventricular contributions to right ventricular systolic function. Progr Cardiovasc Dis 1998; 40: 289-308.

59 Lazar JM, Flores AR, Grandis DJ, et al. Effects of chronic right ventricular pressure overload on left ventricular diastolic function. Am J Cardiol 1993; 72: 1179-1182.

60 Belenkie I, Horne SG, Dani R, et al. Effects of aortic constriction during experimental acute right ventricular pressure loading. Further insights into diastolic and systolic ventricular interaction. Circulation 1995; 92: 546-554.

61 Damiano RJ Jr, La Follette P Jr, Cox JL, et al. Significant left ventricular contribution to right ventricular systolic function. Am J Physiol 1991; 261: H1514-H1524.

62 Marcus JT, Gan CT, Zwanenburg JJ, et al. Interventricular mechanical asynchrony in pulmonary arterial hypertension: left-to-right delay in peak shortening is related to right ventricular overload and left ventricular underfilling. J Am Coll Cardiol 2008; 51: 750-757.

63 Huez S, Faoro V, Vachiery JL, et al. Images in cardiovascular medicine. High-altitude-induced right-heart failure. Circulation 2007; 115: e308-e309.

64 Smith BC, Dobson G, Dawson D, et al. Three-dimensional speckle tracking of the right ventricle: toward optimal quantification of right ventricular dysfunction in pulmonary hypertension. J Am Coll Cardiol 2014; 64: 41-51. 